\title{
Dynamics of activity fronts in a continuum mean field model of cortex
}

\author{
Helmut Schmidt ${ }^{1}$ Ingo Bojak ${ }^{2 *}$, Stephen Coombes ${ }^{1}$ \\ From Nineteenth Annual Computational Neuroscience Meeting: CNS*2010 \\ San Antonio, TX, USA. 24-30 July 2010
}

The functional organization of cortex appears to be roughly columnar, with the laminar sub-structure of each column organizing its micro-circuitry. These columns tessellate the two-dimensional cortical sheet with high density, e.g., $2,000 \mathrm{~cm}^{2}$ of human cortex contain $10^{5}$ to $10^{6}$ macrocolumns, comprising about $10^{5}$ neurons each. Continuum mean field models (cMFMs) describe the mean activity of such columns by approximating the cortical sheet as continuous excitable medium [1]. cMFMs can generate rich patterns of emergent spatiotemporal activity [2]. This has been used to understand phenomena from visual hallucinations to the generation of EEG signals. Pattern boundaries are here defined as the interface between low and high states of average neural activity.

cMFMs support travelling patterns as well as the formation of intricate structures, as in Fig. 1. Here we derive equations of motion for the pattern boundaries of a simple cMFM, showing that their normal velocities are driven by Biot-Savart-style interactions. The solutions of these exact, but dimensionally reduced, equations for activity fronts are in excellent numerical agreement with those of the full nonlinear integral equation defining the neural field. A linear stability analysis of the dynamics of the interfaces allows us to understand mechanisms of pattern formation arising from instabilities of spots, fronts, and stripes. We further test our results against partial differential equations equivalent to the original integral equation, c.f. [3], and perform numerical simulations on a large spatial grid that represents a sizable cortical sheet. In particular, we clarify how more realistic firing rates (computed with

\footnotetext{
* Correspondence: i.bojak@donders.ru.nl

${ }^{2}$ Donders Institute for Brain, Cognition and Behaviour, Centre for Neuroscience, Radboud University Nijmegen (Medical Centre), P.O. Box
} 9101 // 126, 6500 HB Nijmegen, The Netherlands

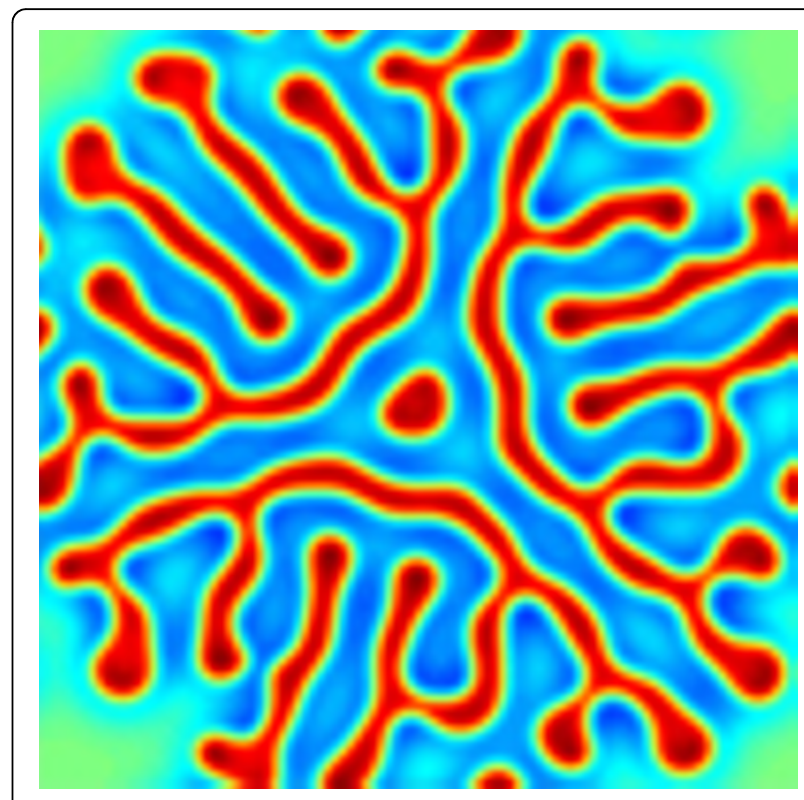

Figure 1 Pattern formation originating in an instable spot. cMFMs support travelling waves that underlie EEG signals; but also spots of localized high firing activity, which have been linked to models of working memory. These spots can become instable and pattern cortex with intricate structures, such as the labyrinthine one shown here. Red color indicates high activity, blue low. The original spot is still visible in the center.

sigmoidal functions instead of Heaviside steps) influence the dynamics of activity fronts.

\section{Conclusions}

Changes of brain activity are often of greater interest than the current state per se. On the cortical sheet, twodimensional patterns can be defined by boundaries between high and low states of activity, and their dynamics can be specified by tracking the evolution of these interfaces. Using a simple cMFM, we show here 
that one can describe the motion of activity fronts with equations of reduced complexity, which nevertheless reproduce the observed dynamics faithfully. This improves our ability to study pattern formation and suggests more generally that modelling the interfaces of patterns, rather than the patterns themselves, may lead to novel, efficient descriptions of brain activity.

\section{Author details}

'School of Mathematical Sciences, University of Nottingham, Nottingham NG7 2RD, UK. ${ }^{2}$ Donders Institute for Brain, Cognition and Behaviour, Centre for Neuroscience, Radboud University Nijmegen (Medical Centre), P.O. Box 9101 // 126, 6500 HB Nijmegen, The Netherlands.

Published: 20 July 2010

\section{References}

1. Coombes S: Large-scale neural dynamics: Simple and complex. Neuroimage 2010, doi:10.1016/.neuroimage.2010.01.045

2. Coombes S, Venkov NA, Shiau L, Bojak I, Liley DTJ, Laing CR: Modeling electrocortical activity through improved local approximations of integral neural field equations. Phys Rev E Stat Nonlin Soft Matter Phys 2007, 76:051901.

3. Bojak I, Liley DTJ: Axonal velocity distributions in neural field equations. PLOS Comput Biol 6:e1000653.

\section{doi:10.1186/1471-2202-11-S1-P28}

Cite this article as: Schmidt et al:: Dynamics of activity fronts in a continuum mean field model of cortex. BMC Neuroscience 2010 11(Suppl 1):P28.

\section{Submit your next manuscript to BioMed Central} and take full advantage of:

- Convenient online submission

- Thorough peer review

- No space constraints or color figure charges

- Immediate publication on acceptance

- Inclusion in PubMed, CAS, Scopus and Google Scholar

- Research which is freely available for redistribution

Submit your manuscript at www.biomedcentral.com/submit 